\title{
I- Function and $\overline{\mathbf{H}}$-Function Associated With Double Integral
}

\author{
Ashok Singh Shekhawat ${ }^{*}$, Rakeshwar Purohit ${ }^{* *}$ And Jyoti Shaktawat ${ }^{* * *}$ \\ *Department of Mathematics, Regional college for Education, Research and Technology, \\ Jaipur, Rajasthan (India) \\ **Department of Mathematics and Statistics, University College of Science, Mohan Lal Sukhadia University, \\ Udaipur, Rajasthan (India) \\ **** Department of Mathematics, Kautilya Institute of Technology and Engineering, \\ Jaipur, Rajasthan (India)
}

\begin{abstract}
The object of this paper is to discuss certain integral properties of a $I$-function and $\overline{\mathrm{H}}$-function, proposed by Inayat-Hussain which contain a certain class of Feynman integrals, the exact partition of a Gaussian model in Statistical Mechanics and several other functions as its particular cases. During the course of finding, we establish certain new double integral relation pertaining to a product involving I function and $\overline{\mathrm{H}}$ function. These double integral relations are unified in nature and act as a key formulae from which we can obtain as their special case, double integral relations concerning a large number of simple special functions. For the sake of illustration, we record here some special cases of our main results which are also new and of interest by themselves. All the result which are established in this paper are basic in nature and are likely to find useful applications in several fields notably electrical network, probability theory and statistical mechanics.
\end{abstract}

Key words. I function, $\overline{\mathrm{H}}$-function, Hermite polynomials, Laguerre polynomials.

\section{Introduction}

The $\overline{\mathrm{H}}$-function [6] is a new generalization of the well known Fox's H-function [4]. The $\overline{\mathrm{H}}$-function pertains the exact partition function of the Gaussian model in statistical mechanics, functions useful in testing hypothesis and several others as its particular cases. The conventional formulation may fail pertaining to the domain of quantum cosmology but Feynman path integrals apply [10,11]. Feynman integral are useful in the study and development of simple and multiple variable hypergeometric series which in turn are useful in statistical mechanics.

The I-function defined as

$$
\begin{aligned}
& \mathrm{I}[\mathrm{z}]=\mathrm{I}\left[\mathrm{z} \mid \begin{array}{l}
\left(\mathrm{a}_{\mathrm{j}}, \alpha_{\mathrm{j}}\right),\left(\mathrm{a}_{\mathrm{ji}}, \alpha_{\mathrm{ji}}\right) \\
\left.\left(\mathrm{b}_{\mathrm{j}}, \beta_{\mathrm{j}}\right), \mathrm{b}_{\mathrm{ji}}, \beta_{\mathrm{ji}}\right)
\end{array}\right] \\
& =\mathrm{I}_{\mathrm{p}_{\mathrm{i}}, \mathrm{q}_{\mathrm{i}}: \ell}^{\mathrm{m}, \mathrm{n}}\left[\mathrm{z}\left[\begin{array}{l}
\left\{\left(\mathrm{a}_{\mathrm{j}}, \alpha_{\mathrm{j}}\right)_{1, \mathrm{n}}\right\},\left\{\left(\mathrm{a}_{\mathrm{ji}}, \alpha_{\mathrm{ji}}\right)_{\mathrm{n}+1, \mathrm{p}_{\mathrm{i}}}\right. \\
\left\{\left(\mathrm{b}_{\mathrm{j}}, \beta_{\mathrm{j}}\right)_{1, \mathrm{~m}}\right\},\left\{\left(\mathrm{b}_{\mathrm{ji}}, \beta_{\mathrm{ji}}\right)_{\left.\mathrm{m}+1, \mathrm{q}_{\mathrm{i}}\right\}}\right\}
\end{array} \mid\right]\right. \\
& =\frac{1}{2 \pi} \oint \theta(\mathrm{s}) \mathrm{z}^{\mathrm{s}} \mathrm{ds}
\end{aligned}
$$

where

$$
\theta(s)=\frac{\prod_{j=1}^{m} \Gamma\left(b_{j}-\beta_{j} s\right) \prod_{j=1}^{n} \Gamma\left(1-a_{j}+\alpha_{j} s\right)}{\sum_{i=1}^{\ell}\left\{\prod_{j=m+1}^{q_{i}} \Gamma\left(1-b_{j i}+\beta_{j i} s\right) \prod_{j=n+1}^{p_{i}} \Gamma\left(a_{j i}-\alpha_{j i} s\right)\right\}}
$$

The $\overline{\mathrm{H}}$-function will be defined and represent as given in [1]

$$
\bar{H}_{P, Q}^{M, N}[x]=\bar{H}_{P, Q}^{M, N}\left[x \mid \begin{array}{l}
\left(a_{j}, \alpha_{j} ; A_{j}\right)_{1, N},\left(a_{j}, \alpha_{j}\right)_{N+1, P} \\
\left(b_{j}, \beta_{j}\right)_{1, M},\left(b_{j}, \beta_{j}\right)_{M+1, Q}
\end{array}\right]=\frac{1}{2 \pi i} \int_{-i \infty}^{i \infty} \bar{\phi}(\xi) x^{\xi} d \xi
$$

where 


$$
\bar{\phi}(\xi)=\frac{\prod_{j=1}^{M} \Gamma\left(b_{j}-\beta_{j} \xi\right) \prod_{j=1}^{N}\left\{\Gamma\left(1-a_{j}+\alpha_{j} \xi\right)\right\} A_{j}}{\prod_{j=M+1}^{Q}\left\{\left(\Gamma 1-b_{j}+\beta \beta_{j}\right) \prod_{j=N+1}^{P} \Gamma\left(a_{j}-\alpha_{j} \xi\right)\right.}
$$

which contains fractional powers of some of the gamma functions. Here

$a_{j}(j=1, \ldots, P)$ and $b_{j}(j=$ $1, \ldots, Q)$ are complex parameters, $a_{j} \geq 0(j=1, \ldots, P), \beta_{j} \geq 0(j=1, \ldots, Q)$ (not all zero simultaneously and the exponents $A_{j}(j=1, \ldots, N)$ and $\quad B_{j}(j=M+1, \ldots, Q)$ can take on non-integer values. The contour in (1.2) is imaginary axis $\mathrm{R}(\xi)=0$. It is suitably indented in order to avoid the singularities of the gamma functions and to keep those singularities on appropriate side. Again for $\quad A_{j}(j=1, \ldots, N)$ not an integer, the poles of the gamma function of the numerator in (1.3) are converted to branch points. However, a long as there is no coincidence of pole from any $\Gamma\left(b_{j}-\beta_{j} \xi\right)(j=1, \ldots, M)$ and $\Gamma\left(1-a_{j}+\alpha_{j} \xi\right)(j=1, \ldots, N)$ pair, the branch cuts can be chosen so that the path of integration can be distorted in the useful manner. For the sake of brevity

$$
\mathrm{T}=\sum_{\mathrm{j}=1}^{\mathrm{M}} \beta_{j}+\sum_{\mathrm{j}=1}^{\mathrm{N}} \mathrm{A}_{\mathrm{j}} \alpha_{j}-\sum_{\mathrm{j}=\mathrm{M}+1}^{\mathrm{Q}} \mathrm{B}_{\mathrm{j}} \beta_{\mathrm{j}}-\sum_{\mathrm{j}=\mathrm{N}+1}^{\mathrm{P}} \alpha_{\mathrm{j}}>0
$$

\section{Main Result}

We will obtain the following result:

(A)

$$
\begin{aligned}
& \int_{0}^{1} \int_{0}^{1}\left(\frac{1-x}{1-x y} v y\right)^{\alpha}\left(\frac{1-y}{1-x y}\right)^{\beta} \frac{1-x y}{(1-x)(1-y)} \mathrm{I}\left[\frac{1-x}{1-x y} v y\right] \bar{H}_{P, Q}^{M, N}\left[\frac{1-y v}{1-x y}\right] d x d y \\
& \left.=\Gamma \alpha+s I\left[v \mid \begin{array}{c}
\left(a_{j}, \alpha_{j}\right),\left(a_{j i}, \alpha_{j i}\right) \\
\left(b_{j}, \beta_{j}\right),\left(b_{j i}, \beta_{j i}\right)
\end{array}\right] \bar{H}_{P+1, Q+1}^{M, N+1}\left[\begin{array}{c}
(1-\beta: 1),\left(a_{j}, \alpha_{j} ; A_{j}\right)_{1, N},\left(a_{j}, \alpha_{j}\right)_{N+1, P} \\
\left(b_{j}, \beta_{j}\right)_{1, M},\left(b_{j}, \beta_{j}: B_{j}\right)_{M+1, Q},(1-k-\alpha-\beta: 1)
\end{array}\right] v\right]
\end{aligned}
$$

provided that $\mathrm{R}\left[\alpha+\beta+\mathrm{b}_{\mathrm{j}} / \beta_{\mathrm{j}}\right]>0,|\arg \mathrm{V}|<\frac{1}{2} \mathrm{~T} \pi$,

Proof. We have

$$
\begin{aligned}
& I\left[\frac{1-x}{1-x y} v y\right] \bar{H}_{P, Q}^{M, N}\left[\frac{1-y)}{1-x y} v\right]=\frac{1}{2 \pi} \oint \frac{\prod_{j=1}^{m} \Gamma\left(b_{j}-\beta_{j} s\right) \prod_{j=1}^{n} \Gamma 1-a_{j}+\alpha_{j} s}{\sum_{i=1}^{\ell}\left\{\prod_{j=m+1}^{q_{i}} \Gamma\left(1-b_{j i}+\beta_{j i} s\right) \prod_{j=n+1}^{p_{i}} \Gamma\left(a_{j i}-\alpha_{j i} s\right)\right\}} \\
& \left(\frac{1-x}{1-x y} v y\right)^{s} \mathrm{ds} \frac{1}{2 \pi \mathrm{i}} \int_{-i \infty}^{\mathrm{i} \infty} \frac{\prod_{j=1}^{\mathrm{M}} \Gamma\left(\mathrm{b}_{\mathrm{j}}-\beta_{j} \xi\right) \prod_{j=1}^{\mathrm{N}}\left\{\Gamma\left(1-\mathrm{a}_{\mathrm{j}}+\alpha_{j} \xi\right)\right\} \mathrm{A}_{\mathrm{j}}}{\prod_{\mathrm{j}=\mathrm{M}+1}^{\mathrm{Q}}\left\{\Gamma\left(1-\mathrm{b}_{\mathrm{j}}+\beta_{\mathrm{j}} \xi\right)\right\} \beta_{\mathrm{j}} \prod_{\mathrm{j}=\mathrm{N}+1}^{\mathrm{P}} \Gamma\left(\mathrm{a}_{\mathrm{j}}-\alpha_{\mathrm{j}} \xi\right)}\left[\frac{1-\mathrm{y}}{1-\mathrm{xy}} \mathrm{v}\right]^{\xi} \mathrm{d} \xi
\end{aligned}
$$

Multiplying both sides of (2.2) by $\left[\frac{1-x}{1-x y} y\right]^{\alpha}\left[\frac{1-y}{1-x y}\right]^{\beta}\left[\frac{1-x y}{(1-x)(1-y)}\right]$

and integration with respect to $\mathrm{x}$ and $\mathrm{y}$ between 0 and 1 for both the variable and making a use of a known result [2, p.145],we get the required result (2.1) after a little simplification 
(B) $\quad \int_{0}^{\infty} \int_{0}^{\infty} \phi(\mathrm{u}+\mathrm{v}) \mathrm{v}^{\beta-1} \mathrm{u}^{\alpha-1} \mathrm{I}(\mathrm{u}) \overline{\mathrm{H}}_{\mathrm{P}, \mathrm{Q}}^{\mathrm{M}, \mathrm{N}}[\mathrm{v}] \mathrm{dudv}$

$$
\begin{aligned}
& \left.=\Gamma \alpha+s \mathrm{I}\left[\mathrm{z} \mid \begin{array}{c}
\left(a_{j}, \alpha_{j}\right),\left(a_{j i}, \alpha_{j i}\right) \\
\left(b_{j}, \beta_{j}\right),\left(b_{j i}, \beta_{j i}\right)
\end{array}\right)\right] \int_{0}^{\infty} \phi(z) z^{\alpha+\beta-1} d z \\
& . \bar{H}_{P+1, Q+1}^{M, N+1}\left[\begin{array}{l}
(1-\beta: 1),\left(a_{j}, \alpha_{j} ; A_{j}\right)_{1, N},\left(a_{j}, \alpha_{j}\right)_{N+1, P} \\
\left(b_{j}, \beta_{j}\right)_{1, M},\left(b_{j}, \beta_{j}: B_{j}\right)_{M+1, Q},(1-s-\alpha-\beta: 1)
\end{array} \mid z\right] d z
\end{aligned}
$$

provided that $R\left(\alpha+\beta+b_{j} / \beta_{j}\right)>0$.

Proof. Using (1.1) and (1.2), we have

$$
\begin{aligned}
& I(u) \bar{H}_{P, Q}^{M, N}[v]=\frac{1}{2 \pi} \int \frac{\prod_{j=1}^{m} \Gamma\left(b_{j}-\beta_{j} s\right) \prod_{j=1}^{n} \Gamma 1-a_{j}+\alpha_{j} s}{\sum_{i=1}^{\ell}\left\{\prod_{j=m+1}^{q_{i}} \Gamma\left(1-b_{j}+\beta_{j i} s\right) \prod_{j=n+1}^{p_{i}} \Gamma\left(a_{j i}-\alpha_{j i} s\right)\right\}} u^{s} d s \\
& \frac{1}{2 \pi \mathrm{i}} \int_{-\mathrm{i} \infty}^{\mathrm{i} \infty} \frac{\prod_{\mathrm{j}=1}^{\mathrm{M}} \Gamma\left(\mathrm{b}_{\mathrm{j}}-\beta_{\mathrm{j}} \xi\right) \prod_{\mathrm{j}=1}^{\mathrm{N}}\left\{\Gamma\left(1-\mathrm{a}_{\mathrm{j}}+\alpha_{\mathrm{j}} \xi\right)\right\} \mathrm{A}_{\mathrm{j}}}{\prod_{\mathrm{j}=\mathrm{M}+1}^{\mathrm{Q}}\left\{\Gamma\left(1-\mathrm{b}_{\mathrm{j}}+\beta_{\mathrm{j}} \xi\right)\right\} \beta_{\mathrm{j}} \prod_{\mathrm{j}=\mathrm{N}+1}^{\mathrm{P}} \Gamma\left(\mathrm{a}_{\mathrm{j}}-\alpha_{\mathrm{j}} \xi\right)} \mathrm{v} \xi
\end{aligned}
$$

Multiplying both side by $\phi(\mathrm{u}+\mathrm{v}) \mathrm{v}^{\beta-1} \mathrm{u}^{\alpha-1}$ and integrating with respect to $\mathrm{u}$ and $\mathrm{v}$ between 0 and $\infty$ for both the variable and make a use of a known result [2, p.177], we get the required result. Letting $\phi(\mathrm{z})=\mathrm{e}^{-\mathrm{pz}}$ in (2.3), we get the particular case after simplification

(c)

$$
\begin{aligned}
& \int_{0}^{1} \int_{0}^{1} \mathrm{f}(\mathrm{uv})(1-\mathrm{u})^{\alpha-1}(1-\mathrm{v})^{\beta-1} \mathrm{v}^{\alpha} \mathrm{I}\left[(\mathrm{v}(1-\mathrm{u})] \overline{\mathrm{H}}_{\mathrm{P}, \mathrm{Q}}^{\mathrm{M}, \mathrm{N}}[1-\mathrm{v}] \mathrm{du} \mathrm{dv}\right. \\
& \left.=\Gamma \alpha+\mathrm{II}\left[(1-\mathrm{z}) \mid \begin{array}{l}
\left(\mathrm{a}_{\mathrm{j}}, \alpha_{\mathrm{j}}\right),\left(\mathrm{a}_{\mathrm{ji}}, \alpha_{\mathrm{ji}}\right) \\
\left(\mathrm{b}_{\mathrm{j}}, \beta_{\mathrm{j}}\right),\left(\mathrm{b}_{\mathrm{ji}}, \beta_{\mathrm{ji}}\right)
\end{array}\right)\right] \int_{0}^{1} \mathrm{f}(\mathrm{z})(1-\mathrm{z})^{\alpha+\mathrm{s}+\beta-1} \\
& \overline{\mathrm{H}}_{\mathrm{P}+1, \mathrm{Q}+1}^{\mathrm{M}, \mathrm{N}+1}\left[\begin{array}{c}
(1-\beta: 1),\left(\mathrm{a}_{\mathrm{j}}, \alpha_{\mathrm{j}} ; \mathrm{A}_{\mathrm{j}}\right)_{1, \mathrm{~N}},\left(\mathrm{a}_{\mathrm{j}}, \alpha_{\mathrm{j}}\right)_{\mathrm{N}+1, \mathrm{P}} \\
\left(\mathrm{b}_{\mathrm{j}}, \beta_{\mathrm{j}}\right)_{1, \mathrm{M}},\left(\mathrm{b}_{\mathrm{j}}, \beta_{\mathrm{j}}: \mathrm{B}_{\mathrm{j}}\right)_{\mathrm{M}+1, \mathrm{Q}},(1-\mathrm{s}-\alpha-\beta: 1)
\end{array} \mid(1-\mathrm{z})\right] \mathrm{dz}
\end{aligned}
$$

provided that $\mathrm{R}(\alpha)>0, \mathrm{R}(\beta)>0$.

Proof. Using equation (1.1) and (1.2), we have

$$
I[v(1-u)] \bar{H}_{P, Q}^{M, N}[1-v]=\frac{1}{2 \pi} \int \frac{\prod_{j=1}^{m} \Gamma\left(b_{j}-\beta_{j} s\right) \prod_{j=1}^{n} \Gamma 1-a_{j}+\alpha_{j} s}{\sum_{i=1}^{\ell}\left\{\prod_{j=m+1}^{q_{i}} \Gamma\left(1-b_{j}+\beta_{j i} s\right) \prod_{j=n+1}^{p_{i}} \Gamma\left(a_{j i}-\alpha_{j i} s\right)\right\}} v^{s}(1-u)^{s} d s
$$




$$
\frac{1}{2 \pi \mathrm{i}} \int_{-\mathrm{i} \infty}^{\mathrm{i} \infty} \frac{\prod_{\mathrm{j}=1}^{\mathrm{M}} \Gamma\left(\mathrm{b}_{\mathrm{j}}-\beta_{\mathrm{j}} \xi\right) \prod_{\mathrm{j}=1}^{\mathrm{N}}\left\{\Gamma\left(1-\mathrm{a}_{\mathrm{j}}+\alpha_{\mathrm{j}} \xi\right)\right\} \mathrm{A}_{\mathrm{j}}}{\prod_{\mathrm{j}=\mathrm{M}+1}^{\mathrm{Q}}\left\{\Gamma\left(1-\mathrm{b}_{\mathrm{j}}+\beta_{\mathrm{j}} \xi\right)\right\} \beta_{\mathrm{j}} \prod_{\mathrm{j}=\mathrm{N}+1}^{\mathrm{P}} \Gamma\left(\mathrm{a}_{\mathrm{j}}-\alpha_{\mathrm{j}} \xi\right)}(1-\mathrm{v})^{\xi} \mathrm{d} \xi
$$

Multiplying both side of (2.6) by $\mathrm{f}(\mathrm{uv})(1-\mathrm{u})^{\alpha-1}(1-\mathrm{v})^{\beta-1} \mathrm{v}^{\alpha}$ and integrating with respect to $\mathrm{u}$ and $\mathrm{v}$ between 0 and 1 for both the variable and use of result [2, p.243] and by further simplification, we get the result (2.5).

Letting $f(z)=z^{\beta-1}$ in (2.5), we get the particular result after simplification.

\section{Particular Case}

$$
\text { Taking } \mathrm{I}\left[\frac{1-\mathrm{x}}{1-\mathrm{xy}} \mathrm{vy}\right] \rightarrow \mathrm{S}_{\mathrm{n}}^{\mathrm{m}}\left[\frac{1-\mathrm{x}}{1-\mathrm{xy}} \mathrm{vy}\right]
$$

The result in (2.1), (2.2) and (2.3) reduces to the known result after a slight simplification obtained by Chaurasia and Shekhawat [2].

\section{Acknowledgement.}

The authors are grateful to Professor H.M. Srivastava, University of Victoria, Canada for his kind help and valuable suggestions in the preparation of this paper.

\section{References}

[1]. R.G. Buschman and H.M. Srivastava, The $\overline{\mathrm{H}}$-function associated with a certain class of Feynman integrals, J. Phys. A: Math. Gen.. 23 (1990), 4707-4710

[2]. V.B.L. Chaurasia and Ashok Singh Shekhawat, Some integral properties of a general class of polynomials associated with Feynman integrals, Bull. Malaysian Math. Sc. Soc. (2) (28) (2) (2005), 183-189.

[3]. J. Edewards, A Treatise on integral calculus, Chelsea Pub. Co., 2 (1922).

[4]. C. Fox, The G and H-functions as Symmetrical Fourier kernels, Trans. Amer. Math. Soc. 98 (1961), 395-429.

[5]. C. Grosche and F. Steiner, Hand Book of Feynman Path integrals, Springer Tracts in Modern Physics Vol.145, Springer-Verlag Berlin Heidelberg, New York, 1998.

[6]. A.A. Inayat-Hussain, New properties of hypergeometric series derivable from Feynman integrals : I. Transformation and reduction formulae, J. Phys. A: Math. Gen. 20 (1987), 4109-4117.

[7]. A.A. Inayat-Hussain, New properties of hypergeometric series derivable from Feynman integrals :II. A generalization of the Hfunction, J. Phys. A: Math. Gen. 20 (1987), 4119-4128.

[8]. Saxena, R.K., On fractional integer operators, Math. Zeitschr, 96 (1967), 288-291

[9]. Saxena, R.K. and Gupta, N., Some Abelian theorems for distributional H-function Transformation, Indian J. Pure Appl. Math. 25(8) (1994), 869-879.

[10]. H.M. Srivastava, A contour integral involving Fox's H-function, Indian J. Math. 14 (1972), 1-6.

[11]. H.M. Srivastava and N.P. Singh, The integration of certain products of Multivariable H-function with a general class of Polynomials, Rend. Circ. Mat. Palermo 2(32) (1983), 157-187.

[12]. C. Szego, Orthogonal polynomials, Amer. Math. Soc. Colloq. Publ. 23 Fourth edition, Amer. Math. Soc. Providence, Rhode Island (1975). 